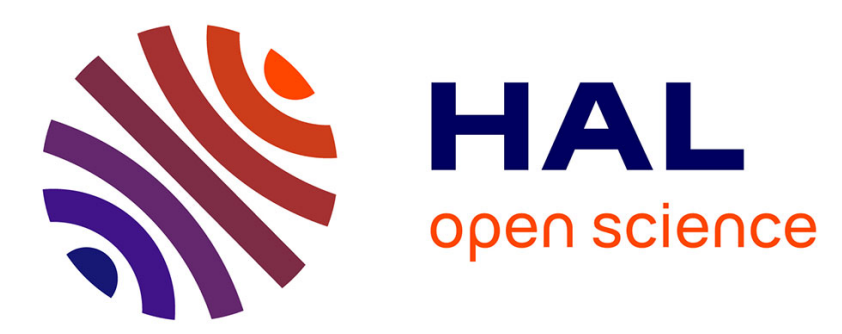

\title{
Folie pubertaire et sexualité diabolique dans les débuts de la psychanalyse
}

Florian Houssier, Angélique Christaki

\section{To cite this version:}

Florian Houssier, Angélique Christaki. Folie pubertaire et sexualité diabolique dans les débuts de la psychanalyse. Topique - Revue freudienne, 2016, 134 (1), 10.3917/top.134.0157 . hal-01423200

\section{HAL Id: hal-01423200 \\ https://hal.science/hal-01423200}

Submitted on 28 Dec 2016

HAL is a multi-disciplinary open access archive for the deposit and dissemination of scientific research documents, whether they are published or not. The documents may come from teaching and research institutions in France or abroad, or from public or private research centers.
L'archive ouverte pluridisciplinaire HAL, est destinée au dépôt et à la diffusion de documents scientifiques de niveau recherche, publiés ou non, émanant des établissements d'enseignement et de recherche français ou étrangers, des laboratoires publics ou privés. 


\section{Folie pubertaire et sexualité diabolique dans les débuts de la psychanalyse}

Résumé : Dans cet article, nous repartons de la période des premiers textes de Freud pour montrer comment certaines vignettes cliniques ouvrent sur l'articulation entre sexualité adolescente et symbolique religieuse en rapport avec une symptomatologie névrotique, le plus souvent sur un versant hystérique ; ces jeunes patients, peu connus ou retravaillés dans la littérature psychanalytique, montrent comment la pulsionnalité effractive de l'adolescence a des effets cliniques divers, notamment dans l'intensification du surmoi et les symptômes à caractère hallucinatoires qui en dérivent. Notre démarche, en indiquant comment Freud tente de dédiaboliser la sexualité hystérique concernant nombre de jeunes gens, ouvre un débat autour des débuts de la psychanalyse. Cela nous amène à poser l'hypothèse selon laquelle certains troubles de l'adolescence, en passant par les traductions dans le champ symbolique du religieux, ont constitué un facteur décisif dans la découverte de la psychanalyse.

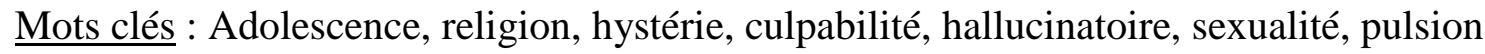


La possession démoniaque fut pendant des siècles une étiologie populaire concernant un certain nombre d'expressions psychopathologiques. Les délires de possession ou encore mystique constituent par exemple autant d'indications sémiologiques ayant trait à la clinique des hallucinations et donnent à entendre l'empreinte des croyances populaires religieuses dans les manuels de psychiatrie de l'époque. La religion, en tant qu'au service d'un refoulement puissant en rapport avec la sexualité, a participé à l'occultation de la place et de la fonction de la sexualité dans la constitution de l'être humain et dans sa société d'appartenance. A travers ce prisme, Freud pose la religion comme un des moyens dont dispose la civilisation pour contraindre la satisfaction pulsionnelle avec des effets parfois à double tranchant tant sur le plan individuel que collectif. Le sentiment religieux lié à la croyance plonge sa source dans la détresse de l'homme non seulement face à la nature et à ses éléments mais aussi face à la sexualité, nature et sexualité se rejoignant pour Freud dans la même étoffe, par opposition à ce que représente le domaine de la culture. La conception d'un corps possédé par le diable qui en découle est certes une interprétation étayée sur un déplacement pour appréhender certaines expressions psychopathologiques; elle n'est cependant pas sans fondements car elle porte la marque de la violence avec laquelle la pulsion sexuelle peut s'emparer du corps et du sujet. A cet égard, dédiaboliser la sexualité afin de la mettre au centre de l'étiologie des névroses fut dans un premier temps l'essentiel de la démarche freudienne. Sur un plan plus personnel, rappelons quelques éléments épars liés à son adolescence, au cours de laquelle il se définit déjà comme un athée néanmoins sensible aux fêtes religieuses (Freud, 1971-1881). Associant à partir du rêve des trois parques, Freud (1900) se souvient que le premier roman ${ }^{1}$ qu'il a lu, probablement à treize ans, met en scène un jeune moine, Philammon, en proie à des désirs incestueux envers sa sœur. Plus encore, le fantasme d'être le Christ que Freud (1908) relèvera comme typique de l'adolescence trouve un écho dans l'identification de Freud au Christ crucifié lorsqu'il raconte à Fliess, dans la lettre du 23 mars 1900, la période dépressive qu'il traverse : «Il n'y a pas le moindre remède à ce qui m'oppresse ; c'est ma croix, je dois la porter, et Dieu sait que mon dos pour s'y faire s'est notablement courbé » (Freud, 2006, p. 513). Freud mêle également le religieux à l'intime lorsqu'il donne à Fliess le surnom de «Démon », ou encore, à sa fille Anna, de « diable noir », bien avant « Antigone » (Houssier, 2010).

Dans le présent article, c'est avant tout à partir des références chrétiennes et non juives que

\footnotetext{
${ }^{1}$ Hypatia, ou ennemis nouveaux à visage ancien, de Charles Kingsley (1853).
} 
nous abordons cette thématique; nous repartons de la période des premiers textes de Freud pour montrer comment certaines vignettes cliniques ouvrent sur l'articulation entre sexualité génitale et symbolique religieuse en rapport avec une symptomatologie névrotique, le plus souvent sur un versant hystérique ; ces jeunes patients, peu connus ou retravaillés dans la littérature psychanalytique, ont pourtant donné à voir et à entendre la virulence du sexuel, au moment du début d'adolescence en particulier. La lettre à Fliess rédigée le 30 janvier 1899 (Freud, 2006, p. 435) fait ainsi écho avec de nombreuses occurrences cliniques insérées dans l'ouvrage qu'il achève cette année là (Freud, 1900) : «De plus en plus la puberté vient occuper une position centrale, la clé de la fantaisie se confirme ». Le débat s'ouvre et se déplie d'une part autour des débuts de la psychanalyse, et d'autre part autour de l'hypothèse selon laquelle certains troubles de l'adolescence, en passant par les traductions dans le champ symbolique du religieux, ont constitué un facteur décisif dans la découverte de la psychanalyse.

\section{$\underline{\text { Sexualité adolescente et stigmates hystériques }}$}

Dans la période pré-analytique, l'association entre sexualité post-pubère et l'hystérie constitue un des fils rouges de l'investigation freudienne; on a parfois oublié qu'avant de s'intéresser aux jeunes filles/femmes souffrant d'hystérie, c'est l'hystérie masculine qui a intéressé Freud (1886). Dans ce texte inaugural sur l'hystérie, il fait part de son expérience chez Charcot quelques mois auparavant; il suggère une étiologie des troubles qui associe les conditions héréditaires et le transfert de la prédisposition de la mère au fils à la «période de la puberté » (Ibid, p. 43), ce qui indique au passage que, dés le début de son œuvre, il ne réduit pas la puberté au seul événement physiologique. Ajoutons à cela que le cas clinique, issu de la pratique de Charcot, qu'il choisit pour illustrer la question parle d'un jeune homme de dixsept ans traumatisé par une chute provoquant nombre de symptômes, corporels ici avant de devenir progressivement psycho-somatiques. Dans son article consacré plus directement à Charcot (Freud, 1893), il établit un lien entre la croyance dans la possession par le démon et les phénomènes hystériques; sa méthode apparaît alors au grand jour: remplacer les croyances superstitieuses issues du Moyen-Age par une perspective et un vocabulaire scientifique. S'appuyant sur ses maitres tels que Charcot mais aussi Breuer, il soutient l'idée de constituer un autre savoir «pour établir le projet d'une théorie de la névrose qui coïncide avec la conception du Moyen-Age, une fois qu'elle a remplacé le « démon » de la fantaisie 
cléricale, par une formule psychologique » (Ibid, p. 415). Chez le garçon, la problématique hystérique côtoie la folie du doute, l'usage de mots orduriers ou les tics; cette symptomatologie d'allure obsessionnelle est associée aux graves blasphèmes des délires mystiques chez les religieuses, dont l'usage des mots relève alors d'un «érotisme débridé » (Freud, 1892-1893, p. 326). Les névroses hystériques et obsessionnelles, chez le garçon comme chez la fille, ont partie liée avec des «traits démoniaques » (Ibid, p. 327).

Lorsqu'il définit l'hystérie pour un article de commande dans un manuel général de médecine (Freud, 1888), il évoque ce trouble pour l'enfant et l'adulte ; l'adolescence y occupe une place singulière en tant qu'agent provocateur d'une «première éruption de la névrose » (Ibid, p. 106). La fonction éruptive et révélatrice de l'adolescence s'inscrit en filigrane dans ses préoccupations, avec l'usage d'un terme qui intervient comme une figure-pont entre sexualité post-pubère, névrose hystérique et religion : les stigmates. Ce terme utilisé au Moyen-Age pour démontrer la présence d'une possession diabolique revient dans plusieurs articles de la période pré-hypnotique, avant d'être partagé par Freud et Breuer (1895) dans un débat sur les stigmates hystériques. Dans ce texte de 1888 , ce terme est associé à une symptomatologie hystérique «aigüe » reliant «l'âge de jeunesse, à partir de quinze ans » (Ibid, p. 106) à l'hystérie féminine; dans un autre contexte, pour parler d'un cas de «neurasthénie de jeunesse typique ", il se fera un peu plus précis quant à l'étiologie des troubles en évoquant « les errements sexuels habituels de l'époque de la puberté » (1892-1893, p. 318), ce en quoi on peut déduire sans trop se risquer la désignation en creux du rôle de la masturbation. Lorsqu'il aborde le traitement encore rudimentaire de l'hystérie, il évoque le reproche que l'on peut faire à l'éducation de favoriser, dans les classes sociales aisées notamment, les sentiments raffinés et le développement de la sensibilité ; il suggère que les réactions des parents ou thérapeutes face aux personnes jeunes, lorsqu'elles relèvent de l'autorité et de menaces punitives, ne sont pas mauvaises en soi mais découlent de conceptions fausses, parmi lesquelles les conceptions religieuses occupent une place centrale.

Face à la force des croyances dictées par la religion catholique, les enjeux autour de la découverte de la sexualité s'articulent alors avec la masturbation, le premier rapport sexuel, et, ultérieurement, le mariage et la première grossesse. Un exemple clinique montre l'importance à la fois intrapsychique et sociale des enjeux autour de la sexualité génitale ; Freud (1900, p. 223) évoque la situation d'un jeune homme qui rêve qu'il remet son pardessus d'hiver, associé au souvenir d'une dame qui lui a révélé que son dernier enfant devait la vie à un préservatif déchiré. Le préservatif devient alors lié à l'idée d'un pardessus, et s'il arrivait à 
ce célibataire ce que cette femme lui a raconté, ce serait terrible pour lui. Les conséquences sociales de la sexualité génitale sont présentes dans le poids du réel concernant ces jeunes gens pour lesquels, en toute identification (Houssier, 2015), Freud souhaita trouver un moyen plus efficace de contraception (Freud, 2006) tout en défendant la nécessité de libérer leur sexualité avant le mariage. Une conception prise dans les enjeux psycho-pédagogiques (Marty, Houssier, 2007) de son temps, mais une préoccupation qui traverse le temps : soutenir une moindre répression des pulsions face aux prescriptions religieuses à un moment où celles-ci sont encore impliquées dans les conduites morales et sociales à tenir, au risque d'une contention libidinale intenable, notamment au moment de l'adolescence.

\section{$\underline{\text { L'angoisse des jeunes vierges face au premier rapport sexuel }}$}

Le surgissement traumatique des pulsions à l'adolescence est lié à une excitation qui déborde les capacités psychiques de contenance et de mise en sens du sujet, impliquant des sensations corporelles envahissantes; l'intensification pulsionnelle et ses effets confèrent à l'avènement de la sexualité génitale un caractère à la fois désorganisateur et persécutant, parfois traduit en termes religieux par une terminologie qui qualifie ces sensations comme «diaboliques » ou « démoniaques ». Le repérage de certains fantasmes à caractère religieux indique que Freud ne perd pas de vue la place de la métaphore religieuse dans les modalités de constitution et d'expression du symptôme chez ces jeunes patientes ; ce qu'illustre l'identification idéalisante à la Madone dans le court exemple suivant. La figure de la Madone et le fantasme de la parthénogenèse auquel elle renvoie constituent ainsi une figure religieuse de prédilection qui recouvre une sexualité coupable chez les jeunes adolescentes (Freud, 1905 b). «J'eus le premier soupçon de ces associations alors que, médecin d'une clinique psychiatrique, j'observai chez une jeune fille un état confusionnel hallucinatoire aigu qui se révéla être une réaction à un reproche de son fiancé » (Ibid, p. 77).

L'observation clinique des débuts d'adolescence participe clairement à la compréhension de la psychopathologie alors que l'hystérie est au centre de l'intérêt de Freud dans les débuts de la psychanalyse. A ce sujet, Freud (2006, p. 70) écrit une lettre du 30 mai 1893 à W. Fliess : «Dans l'étiologie sexuelle des névroses je vois une bonne possibilité de combler de nouveau une lacune. Je crois comprendre que concernant les névroses d'angoisse des adolescentes, que l'on doit nécessairement considérer comme vierges, et qui n'ont pas été soumises au mésusage (sexuel) il y avait à l'arrière-plan cette horreur pleine de pressentiment vis-à-vis de 
la sexualité, des choses qu'ils avaient vues ou entendues et à moitié comprises, donc une pure étiologie d'affect, mais pourtant de nature sexuelle. » Ces jeunes filles au cour virginal révèlent leurs angoisses devant la première confrontation au monde de la sexualité. Dans ces lettres à Fliess, lorsqu'il évoque le cas clinique, en 1893-1895, d'une jeune femme qui raconte qu'avant le mariage, elle s'est trouvée débordée par des accès d'angoisse quand, dormant à côté de la chambre de ses parents, elle a pu voir son père entrer dans le lit de sa mère et entendu quelque chose qui l'a beaucoup frappée, c'est d'hystérie dont il est généralement question, venant souvent, dans la prose de Freud, recouvrir les conflits de l'adolescence.

A partir des études sur l'hystérie (Freud, Breuer, 1895), Freud établit un lien plus direct entre l'adolescence et la compréhension de l'hystérie, lien soutenu par la découverte du concept d'après-coup qui articule le traumatisme infantile avec le début de l'adolescence d'Emma (Freud, 1895). «Eckstein a une scène où le Diabolus lui pique des aiguilles dans les doigts et pose sur chaque goutte de sang un bonbon », écrit Freud (2006, p. 286) à Fliess le 17 janvier 1897 ; Freud relie ce moment de la cure d'Emma au rôle des épingles écorchant les seins des femmes soumises à l'Inquisition pour leur faire avouer leur commerce avec le diable, c'est-àdire leurs fantasmes.

Comme pour Emma, c'est également dans l'après-coup de cures de patients adultes que les souvenirs d'adolescence remontent à la surface. Au point que, pour le cas de Cäcilie, l'adolescence apparait comme la source du trouble psychogène (Freud, 1900, p. 575) : «Dès 1895, je pus (...) communiquer l'explication d'une première crise d'angoisse hystérique qu'une malade âgée de 40 ans avait eue dans sa quinzième année ».

Dans sa pratique comme dans les théorisations qui en découlent, Freud affirme le caractère choquant des «affaires » sexuelles et l'impact de celles-ci sur l'imagination de jeunes filles chastes. Il suggère la dimension œdipienne des personnes prédestinées à la névrose en parlant des enfants précoces et avides d'affection, excités par des sensations génitales spontanées, une scène de séduction ou la masturbation; avant d'ajouter que certaines influences se font alors valoir, en fixant la tendance amoureuse à un point tel «qu'elle devient, dès l'enfance ou à la puberté seulement, quelque chose de comparable à une attraction sexuelle, quelque chose qui, comme celle-ci, accapare la libido » (1905 b, p. 40).

Découverte de la sexualité génitale et état confusionnel hystérique font partie des éléments essentiels qui traversent ces diverses évocations cliniques. Les désirs sexuels, comme les exemples qui suivent l'illustrent, prennent une teneur hallucinatoire - qu'on ne confondra pas avec une hallucination - à la hauteur de leur intensité et du caractère psychiquement 
désorganisateur de la puberté au début de l'adolescence.

\section{De quelques potentialités hallucinatoires}

Freud (1900) découvre les liens entre la régression qui régit l'inscription des traces mnésiques et les hallucinations dans l'hystérie. Plus particulièrement, les pensées en relation avec les souvenirs réprimés ou inconscients sont celles qui vont acquérir, notamment au début de l'adolescence, une forte teneur hallucinatoire. Freud n'hésite pas à qualifier d'hystérique un adolescent de douze ans, selon le tableau nosologique dominant de cette époque ; ce garçon souffre de troubles d'endormissement lié à la vision des visages verts avec des yeux rouges. Freud relie cette vision au souvenir, quatre ans auparavant, d'un camarade qui se masturbait, comportement que ce jeune adolescent finit par «se reprocher à lui-même maintenant de façon rétrospective » (Ibid, p. 598), indiquant dans son sous-texte que l'adolescence est un révélateur de l'infantile. La mère du patient avait tenu des propos sur le teint verdâtre et les yeux cernés de rouges de ce camarade. Ces commentaires sont associés à d'autres phrases de sa mère, teintées de menaces de castration punissant les pratiques masturbatoires : devenir idiot et mourir précocement. Et comme en écho avec la réalisation de ces menaces, le patient échoue au lycée et craint pour sa vie. L'efficacité de la psychothérapie lui permet de retrouver le sommeil et d'obtenir d'excellents résultats scolaires, sans qu'on puisse en savoir davantage sur ce qui a délivré ce jeune garçon si ce n'est de pouvoir en parler à un adulte, Freud, qui ne porte pas de jugement.

Les visions à caractère hallucinatoire portent la marque d'un sentiment de culpabilité inconscient (Freud, 1923, p. 264) lié à l'activité masturbatoire pendant que l'échec scolaire porte la charge et la réalisation de cette menace redoutée : devenir idiot, qui se substitue ici à un autre fantasme prégnant à l'adolescence, devenir fou.

Si les conflits masturbatoires ont bien pris leur source dans la vie infantile du patient, c'est à l'adolescence qu'ils se potentialisent et s'actualisent, sur fond de menaces maternelles. Le père est l'absent de cette scène, mais pas l'analyste qui étaye par son écoute et probablement ses explications cet adolescent. L'état d'angoisse du garçon indique l'ampleur des transformations auxquelles il est confronté: assumer son identité sexuelle masculine et élaborer la nouvelle donne génitale. C'est aussi consécutivement à l'avènement de la puberté que la temporalité coup/après-coup prend toute sa force. Avant le seuil pubère, l'expression hallucinatoire des formations symptomatiques est difficilement repérable cliniquement à 
cause du caractère particulièrement labile du psychisme infantile (Christaki, 2015 b). En revanche, à l'adolescence, ces conflits psychiques peuvent acquérir un relief hallucinatoire plus marqué indiquant par-là la voie élaborative empruntée. Ainsi, halluciner/déshalluciner le caractère traumatique de la rencontre avec le sexuel peut constituer un cheminement à potentialité symbolisante laissant la place au tissage fantasmatique et à des rêveries à même de soutenir un effet de contenance psychique. Enfin, ce qui serait thérapeutique relèverait ici de la possibilité de ré-ouvrir un espace de pensée et de rêverie sans menace interne persécutrice.

La masturbation est encore au centre de la problématique d'une adolescente de quatorze ans (Freud, 1905 b). Ce cas cité dans deux notes de bas de page développe l'idée de ne pas entériner les négations du patient lorsqu'il est question de secrets liés à la sexualité. Freud évoque la situation de cette jeune fille qui fut traitée par un médecin peu enclin à s'intéresser au rôle de la sexualité dans l'étiologie des troubles hystériques, ici représentés par un symptôme d'appel, des vomissements violents. Lorsque le médecin lui demande si elle a eu par hasard une affaire de cœur, elle s'oppose à l'idée en la relatant avec mépris à sa mère : «Pense donc, ce stupide bonhomme m'a même demandé si j'étais amoureuse » (Ibid, p. 15). Lorsqu'elle fut soignée plus tard par Freud, ce dernier explique qu'elle lui révéla, « mais pas au premier entretien », qu'elle se masturbait depuis longtemps ce qui s'accompagnait de pertes blanches, qu'elle s'était déshabituée de cette pratique, provoquant par son abstinence des tourments liés notamment à un sentiment de culpabilité intense : les malheurs qui arrivaient à sa famille incarnaient pour elle le châtiment divin de son péché. Freud ajoute qu'elle était perturbée par l'histoire d'une tante dont on lui avait caché qu'elle avait eu une grossesse illégitime.

Il reprend ce cas plus loin pour illustrer cette fois l'idée de mots dotés d'une signification plurielle et favorisant certaines formulations inconscientes. Il explique avoir «installé » (Ibid, p. 61) sa patiente dans une pension en compagnie d'une femme qui était sa gardemalade ; celle-ci lui raconta que la jeune fille ne supportait pas sa présence lorsqu'elle la couchait, et que dans le lit, elle se mettait à tousser «bizarrement», seulement dans cette situation là. On pose la question sur cette toux à la jeune fille, qui répondit que sa grand-mère toussait ainsi et qu'on la disait atteinte d'un catarrhe ${ }^{2}$. Elle ne voulait pas être surprise le soir pendant sa toilette, le traitement de son affection somatique relevant d'un équivalent masturbatoire. Il conclut : «Le catarrhe qui, à l'aide de ce mot, avait été déplacé de bas en

\footnotetext{
${ }^{2}$ Un catarrhe est une inflammation des muqueuses (nez, poumons, voies respiratoires).
} 
haut, était même d'une intensité peu commune. » Ce déplacement du haut vers le bas chez l'hystérique, du sexe vers le visage, est également associé, dans la lettre que Freud (2006, p. 433) envoie à Fliess le 16 janvier 1899, à la migraine chez la jeune fille qui intervient pour représenter la défloraison violente, que Freud renvoie à un fantasme masturbatoire concernant les jeunes vierges.

La masturbation est placée au centre de la problématique, avec comme conséquence fantasmée un châtiment divin, punition qui laisse supposer la trace d'un sentiment de culpabilité inconscient accompagnée d'un symptôme somatique. Freud ne fait pas mention des parents ni des effets du rapprochement entre la garde-malade et cette jeune fille. Le caractère bizarre signe l'aspect quasi-fantastique à la fois du vécu d'adolescence et du langage symbolique religieux. Il s'avère ainsi que les débuts d'adolescence passent par une intensification du surmoi, substantialisé par les pensées religieuses face à la charge pulsionnelle, conférant à cette instance une dimension persécutrice.

\section{$\underline{\text { Une sexualité terrifiante et démoniaque }}$}

La clinique de l'enfant, souvent de façon rétroactive, et celle, plus directe, de l'adolescent sont présentes dans les préoccupations de Freud, sans rapport de supériorité de l'une sur l'autre même si Freud garde sa boussole en tête, à savoir retrouver les traces infantiles du conflit psychique. Il cite le cas d'Albert, pour dénoncer l'inanité de la perspective médicale et de ses œillères (Freud, 1900). Cette vignette est issue d'une thèse sur les hallucinations et terreurs nocturnes chez les enfants et adolescents datant de 1881 et rédigé par un médecin, Debacker ; ce dernier relate le cas d'un garçon de treize ans dont le père a été touché par la syphilis, convoquant chez le médecin l'hypothèse d'une débilité héréditaire chez le fils. Freud reprend ce cas à son compte de la sorte : depuis deux ans, Albert est aussi rêveur qu'inquiet, de jour comme de nuit. Ses nuits sont agitées par des terreurs nocturnes et des hallucinations récurrentes. Il relate à leur suite avoir aperçu le diable qui, seul ou accompagné, vient lui crier à tue-tête : «Nous t'avons! Nous t'avons! (Ibid, p. 640), à la suite de quoi il sent une odeur de bitume et de soufre, le feu brûlant la surface de son corps préalablement dépouillé de ses vêtements. Les cris qu'il pousse laissent entendre que ce n'est pas lui, qu'il n'a rien fait, ou encore une supplique pour qu'on le laisse, qu'il ne le fera plus, qu'il n'a jamais fait ça. Un détail est relevé par Freud dans ce récit : Albert ne voulait plus se coucher ni se déshabiller de peur que le feu ne l'atteigne. Envoyé à la campagne, ses troubles ont cessé et, âgé de quinze 
ans, il peut rire de ses anciennes terreurs.

Dans cet après-coup, il évoque ainsi l'intensité de ses angoisses : «Je n'osais pas l'avouer, disait-il depuis, mais j'éprouvais continuellement des picotements et des surexcitations aux parties ; à la fin cela m'énervait tant que plusieurs fois j'ai pensé à me jeter par la fenêtre du dortoir $\gg($ Idem $)$.

Le commentaire de Freud renvoie à la masturbation infantile, aux sévères menaces reçues par l'enfant et à la culpabilité inconsciente qui les accompagne en retour. Il ajoute que «sous la poussée de la puberté » (Ibid, p. 499), la tentation de se masturber à cause des picotements dans ses organes génitaux a refait surface, provoquant un nouveau mouvement de refoulement propice à la résurgence de l'angoisse. Critiquant la tentation biologisante de l'analyse du médecin - le lien entre la syphilis du père et la débilité supposée de l'enfant -, Freud établit de son côté un lien entre la vision du démon et l'influence religieuse dans l'environnement du garçon, comme source d'identification hystérique relative aux représentations culturelles de son temps, tandis que l'effet de seuil pubère ravive les conflits infantiles non résolus. La puberté agit par le corps sur la vie psychique comme un agent de déstabilisation de l'économie libidinale qui, sans trouver de nouvelles voies de dérivation et dans l'attente d'une réalisation plus directe, ne peut que se révéler explosive pour la vie psychique, au risque ici d'un passage à l'acte suicidaire. L'irruption du sexuel génital est donc associé à un persécuteur interne représenté par le démon, renvoyant à un « habillage » de la pulsion.

Le caractère démoniaque de la pulsionnalité sexuelle pubère n'est pas forcément lié aux paroles parentales, il peut tout aussi bien être associé au bain culturel d'une époque, la croyance religieuse impliquant des menaces sexuelles. L'exemple qui suit, celui d'une jeune fille qui se sent secouée dans tout son corps lors d'une consultation médicale de Freud, aurait bien pu être qualifié de possession diabolique, alors qu'il relève avant tout d'une sexualité génitale folle qui s'empare du corps d'une adolescente. De même que les sensations génitales du jeune garçon (les picotements) ou encore le feu qui menaçait de brûler son corps lorsqu'il se trouvait nu, cette jeune fille rend compte de la folie pubertaire dans le sens d'une génitalité insupportable, intolérable parce que pas encore symbolisée, si ce n'est par des angoisses terrifiantes, psychotic like aurait pu écrire A. Green (1990). Revenons à Freud et à une consultation probablement médicale, en compagnie d'un collègue. La jeune fille a un abord «bizarre » (Freud, 1900, p. 673) trouve-t-il, dans le sens où elle ne se présente pas de façon soignée mais laisse pendre un de ses bas tandis qu'un bouton de son corsage est défait. Cette attitude s'accompagne de plaintes concernant des douleurs dans une jambe, ce qu'elle illustre 
en montrant spontanément son mollet. Laissons la prose de Freud parler d'elle-même : «Elle a l'impression d'avoir dans le corps «quelque chose de caché » qui va et vient et la « secoue » tout entière. Souvent alors tout son corps se raidit. » (Idem). Raidir son corps peut aussi être entendu comme une tentative de maitriser son corps face à un spasme orgastique que le raidissement représente dans le même temps par mimétisme.

Freud et son collègue trouvent surprenant que la mère ne comprenne pas le caractère extrêmement séducteur de la jeune fille, dont l'ingénuité seule peut permettre une telle expression de fantasmes sexuels à la façon, commente Freud, d'une rêverie ordinairement inconsciente ; à la différence que ces fantasmes passent par un langage de l'acte qui n'exclut pas la rêverie de désirs mais en limite la seule portée pour se rendre plus explicitement sur le terrain d'une actualisation pré-symbolique. Si Freud relèvera les fantasmes de prostitution des jeunes filles, il ne souligne pas le caractère adolescent de certaines crises hystériques, qui tend à s'estomper une fois le processus adolescent plus conflictualisé s'installe sur un mode secondarisé. Dans ces temps de folie pubertaire, les processus primaires sont au premier plan, mettant en tension la censure comme l'équilibre des défenses : une adolescente exprime un questionnement sur sa sexualité sans mot dire alors que sa mère ne veut rien en savoir. Corps brûlant, corps découvert/exhibé, le corps parle lorsque les mots et leur sens polysémique sont relégués au second plan, en attente de re-signification. Sans même se référer à la scène primitive, cette adolescente montre ce qui l'agit de l'intérieur de son corps pubère : une simulation de rapport sexuel, une intuition de ce qui se passerait si elle couchait avec un homme, mais aussi l'envie d'un orgasme génital à travers le raidissement de son corps.

L'absence de compréhension de la mère laisse infiltrer l'idée d'une complicité de celle-ci, celle qui n'aurait pas protégée sa fille de l'excès d'une excitation sexuelle que la jeune fille tente de scénariser, de figurer par sa mise en scène. La surdité maternelle au moment du réaménagement des identifications sexuelles dans le lien mère-fille ne fait que déchainer la violence du sexuel, donnant à voir la force du questionnement à l'endroit où il n'est pas entendu. Ainsi la violence du questionnement est portée jusqu'au point paroxystique de l'hallucination cénesthésique que le théâtre du corps hystérique donne à voir. On imagine sans peine l'impact d'une telle scène sur deux hommes mûrs pour corroborer l'hypothèse d'une étiologie sexuelle des troubles des jeunes filles, mais sans la penser pour autant reliée à l'entrop pubertaire, traumatique en diable...

\section{$\underline{\text { Haine dans le lien père-fils }}$}


Ce dernier exemple montre la pratique de Freud comme médecin consultant au moment où il cherchait à gagner sa vie et où il se tournait de plus en plus vers la psychanalyse en se dégageant d'une approche médicale raillée pour son aveuglement dans la compréhension des patients. Un autre exemple permet de saisir que, alors qu'il abandonnait l'hypnose mais pas la suggestion, Freud (1900) a reçu un adolescent de quatorze ans souffrant de troubles divers (tics convulsifs, vomissements hystériques, migraines, etc.). Il s'agit du dernier exemple clinique du livre, qui révèle à Freud l'angoisse de castration dans la névrose, ce qu'il appelait pressentait comme un chainon manquant pour compléter sa théorie de la névrose (Anzieu, 1959).

Lui suggérant de fermer les yeux et de laisser venir librement les images ou idées, la réponse ne se fait pas attendre, et elle passe par les images, augurant l'hypothèse d'une régression de l'appareil psychique des idées aux images, la rêverie résultant d'un compromis entre pensée hallucinée et pensée concrète. Les images qui s'imposent au jeune garçon sont celle d'un échiquier de dames et d'une partie avec son oncle. Ces images sont progressivement accompagnées d'expressions métaphoriques liées au jeu: les coups que l'on peut tenter, la vision d'un poignard sur l'échiquier, une faucille, une faux, et enfin un vieux paysan fauchant le gazon devant la maison pourtant éloignée de son père.

Là encore, l'atmosphère familiale était dominée par un climat de menaces, cette fois paternelles. Les conflits de couple, avec un père dur et colérique, aboutirent à un divorce, provoquant la séparation de l'enfant avec sa mère aimée qui fut remplacée par une jeune femme. La signification symbolique donnée par Freud renvoie à la faucille est celle avec laquelle Zeus a émasculé son père Kronos, qui est incarné par l'image du paysan et représente le méchant vieillard qui mange ses enfants et dont Zeus tire une vengeance si peu filiale.

Cette référence à la mythologie n'est pas sans lien avec le religieux quand on sait à quel point Freud a puisé dans la Bible et dans des exemples mythologiques, ou encore si on relit ce que nous avons considéré comme un mythe phylogénétique (Houssier, 2013), l'histoire de la horde primitive qui, dans ses finalités, aboutit à la croyance religieuse à partir du repas totémique au cours duquel le père de cette horde fut mangé (Freud, 1913).

Freud (1900, p. 674) conclut la vignette ci-dessus par ce commentaire : « Le mariage du père est une occasion de lui retourner les reproches et les menaces qu'il fit autrefois à l'enfant parce qu'il jouait avec ses organes génitaux (le jeu de dames, les coups défendus, le poignard meurtrier). Voilà les souvenirs longtemps refoulés et leurs dérivés inconscients qui se sont 
glissés dans la conscience sous forme d'images en apparence vides de sens. » La menace liée à la masturbation reste au cour de l'interprétation de Freud, malgré le caractère typique du fantasme de castration relevé à travers la mythologie. Cette dimension inquiétante dans le lien père-fils relève aussi de la reprise des désirs et angoisses œdipiens, à la lueur de la nouveauté génitale. On peut aussi inférer que le jeune garçon peut séduire la mère comme la belle-mère qui représente à la fois une source de vengeance contre le père mais également une prime d'excitation une fois la puberté advenue. Le début d'adolescence apparait alors comme une source d'excitabilité des conflits infantiles; mais plus encore, si on suit le fil de la réactivation des fantasmes passif-actifs au début de l'adolescence, ce n'est pas seulement les désirs meurtriers qui sont pour la première fois devenus réalisables pour l'adolescent. Ce fantasme, «je suis tué par le père », est retourné sur un mode passif, représenté par le poignard ou le vieux paysan et sa faux mortelle, ce fantasme pouvant désormais devenir réalité. Cette fois, il est possible de tuer le père ou d'être tué par lui, dans une logique paranoïaque du type «lui ou moi » que Freud a exploré dans le récit des origines de l'humanité.

\section{$\underline{\text { L'adolescence à son insu }}$}

Le renoncement aux pulsions sexuelles comme prescription religieuse s'origine dans le surmoi de la communauté civilisée et dans ses exigences idéales; alors que le sentiment de culpabilité qui s'y rattache constitue une variante de l'angoisse face au surmoi, dont la frange inconsciente est identifiée par Freud en tant qu'une des plus puissantes résistances dans l'analyse. Si l'homme d'église auquel s'est adressée l'hystérique moyenâgeuse a prescrit le feu pour elle, l'hystérique écoutée par Freud a participé avec son savoir et son questionnement sur la sexualité à la découverte de l'inconscient comme hypothèse fondatrice de la psychanalyse (Christaki, 2015 a). Parmi ces sujets, on trouve nombre de jeunes gens qu'on appellerait aujourd'hui des adolescents, préoccupés notamment par la découverte d'une sexualité génitale à intégrer à la fois sur les plans somatique et psychique. La clinique de l'adolescence rejoint le positionnement de Freud contre la religion et la médecine, des savoirs ou croyances constitués, dans les éléments qui ont contribué de façon essentielle à la naissance de la psychanalyse.

Ainsi, bien des exemples cliniques relatés par Freud (1905 a) jusqu'au troisième essai sur la théorie de la sexualité portent la trace d'un questionnement insufflé par l'écoute des 
adolescents quand bien même elle n'est pas nommée comme telle par Freud qui le travaille en quelque sorte à son insu, ou de façon ponctuelle ou allusive. Comme l'ont montré ces exemples cliniques, endiguer la vague pulsionnelle liée aux réaménagements identificatoires et aux transformations pubertaires pendant le temps de l'adolescence peut s'avérer une tâche que l'activité fantasmatique peine à prendre en charge. Quand l'activité symbolique et fantasmatique échouent face à cette charge psychique, il advient que d'autres mécanismes psychiques prennent le relais : refoulement, mode de représentance hallucinatoire ou encore recours au langage symbolique de l'acte.

L'activité hallucinatoire, étayant les représentations religieuses notamment lorsqu'il est question de visions, intervient comme une tentative de tissage d'un voile psychique à l'endroit de l'effraction traumatique. La dimension hallucinatoire du symptôme est repérée par Freud comme relief d'une intensité particulière indiquant la charge sexuelle ; cette intensité peut prendre une allure démoniaque indiquant l'actualisation du sexuel pubertaire en vue d'une tentative de névrotisation des conflits (Marty, 2001).

Bibliographie

Anzieu D., L'auto-analyse de Freud et la découverte de la psychanalyse, Paris, PUF, 1959.

Christaki A., "Contorcao histerica no século XXI: elixirs e remedos" in Revista Trivium Est. Intered, VII, 1, 2015 a, p. 30-35.

Christaki A., "Hallucinations multi-sensorielles et clinique infantile" in L'Evolution psychiatrique on line, 2015 b, à paraître.

Freud S., Lettres de jeunesse (1871-1881), Paris, Gallimard, 1990.

Freud S., «De l'hystérie masculine (1886) », in OC, I (1886-1893), Paris, PUF, 2015, p. 3952.

Freud S., Lettres à Wilhelm Fliess (1887-1904), Paris, PUF, 2006.

Freud S., «Hystérie (1888) », in OC, I (1886-1893), Paris, PUF, 2015, p. 91-112.

Freud S., «Un cas de guérison hypnotique, accompagné de remarques sur l'apparition de symptômes hystériques du fait de la “contre-volonté" (1892-1893) », in OC, I (1886-1893), Paris, PUF, 2015, p. 315-329.

Freud S., “Charcot (1893)”, in OC, I (1886-1893), Paris, PUF, 2015, p. 401-416.

Freud S., Breuer J. (1895), Les études sur l'hystérie, Paris, PUF, 1965.

Freud S. (1895), Esquisse d'une psychologie scientifique, in La naissance de la psychanalyse, Paris, PUF, 1956, p. 307-396. 
Freud S., «Sur les souvenirs-écrans (1899)», in Névrose, psychose et perversion, Paris, P.U.F., 1973, p. 113-132.

Freud S. (1900), L'interprétation du rêve P.U.F., 2010.

Freud S., Trois essais sur la théorie de la sexualité, (1905 a) Paris, Gallimard, 1962.

Freud S., «Fragments d'une analyse d'hystérie : Dora (1905 b) », in Cinq psychanalyses, Paris, PUF, 1954, p. 2-91.

Freud S. (1908), in Nunberg H., Federn E. (dir.), Les Premiers Psychanalystes. Minutes de la Société psychanalytique de Vienne, 1908-1910, T. II, Paris, Gallimard, 1978.

Freud S. (1913), Totem et tabou, Paris, Payot, 1947.

Freud S., «Le moi et le ça (1923)», in Essais de psychanalyse, Paris, Payot 1991.

Green A., Point de vue du psychanalyste sur les psychoses à l'adolescence, in Ladame F., Gutton P., Kalogerakis M. (dir.), Psychoses et adolescence, Paris, Masson, 1990, p. 231-244.

Houssier F., L'école d'Anna Freud. Créativité et controverses, Paris, Campagne-Première, 2010.

Houssier F., Meurtres dans la famille, Paris, Dunod, 2013.

Houssier F., «Freud adolescent», in Les Cahiers de l'Herne (dir. R. Perron, S. Missonnier), Freud, 2015, p. 31-37.

Marty F., La violence de l'adolescence: de l'événement traumatique à la névrose de l'adolescence, in Marty F. (dir.), Figures et traitements du traumatisme, Paris, Dunod, 2001, p. 1-13.

Marty F., Houssier, F. (dir.), Eduquer l'adolescent? Pour une pédagogie psychanalytique, Collection Recherches : Les troubles de l'enfance et de l'adolescence, Champ Social Editions, Diffusion Les Belles Lettres, Nîmes, Décembre 2007, 260 pages.

Florian Houssier

Psychologue, Psychanalyste - Président du Collège International de l'Adolescence (CILA) Pr de Psychologie clinique et Psychopathologie, Unité Transversale de Recherches: Psychogenèse et Psychopathologie (UTRPP), Université Paris 13, Villetaneuse, Sorbonne Paris Cité.

Angélique Christaki

Docteur en psychopathologie fondamentale et psychanalyse

Chercheur associé au CRPMS de l'université Paris 7

Psychanalyste - psychologue clinicienne en CMPP-BAPU 